\title{
Modified fenestration technique for the Kawashima operation in a young infant
}

\author{
Dante Picarelli, MD, ${ }^{a}$ José Luis Montenegro, MD, ${ }^{b}$ Serrana Antunez, MD, \\ Walter Perez, MD, ${ }^{\mathrm{c}}$ and Daniel Borbonet, MD, ${ }^{\mathrm{c}}$ Montevideo, Uruguay
}

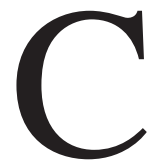

ases involving small infants undergoing the Kawashima operation for left atrial isomerism, single ventricle, and azygos continuation of the inferior vena cava are rare and challenging because of the risk associated with this particular circulation, which is similar to a total cavopulmonary shunt, except for the hepatic flow. ${ }^{1}$

Taking into account the recent encouraging report from Hannan and associates, ${ }^{2}$ we decided to perform a modified fenestrated Kawashima operation, preserving a limited antegrade flow, in a $51 / 2$-month-old infant with severe cyanosis caused by progressive pulmonary stenosis.

\section{Clinical Summary}

With a previous prenatal echocardiographic diagnosis of single double-outlet right ventricle, malposition of the great arteries, common atrium and atrioventricular valve, and azygos continuation of interrupted inferior vena cava, a female neonate was born after 37 weeks' gestation weighing $2760 \mathrm{~g}$. She had mild cyanosis (oxygen saturation of $85 \%$ ) without signs of cardiac failure. Postnatal echocardiography confirmed the diagnosis and revealed a mild subpulmonary stenosis $(23 \mathrm{~mm} \mathrm{Hg}$ gradient) and bilateral superior venae cavae.

After 5 months, cyanosis progressively increased (oxygen saturation of $70 \%$ ) because of severe subpulmonary stenosis, with a Doppler gradient of $70 \mathrm{~mm} \mathrm{Hg}$ confirmed by cardiac catheterization.

To increase the pulmonary artery flow, we decided to perform a fenestrated Kawashima operation, despite the young age of the patient. Intraoperative mean pulmonary artery pressure before the procedure was $14 \mathrm{~mm} \mathrm{Hg}$.

A standard bilateral bidirectional cavopulmonary anastomosis was constructed during normothermic cardiopulmonary bypass. The fenestration technique described by Hannan and associates ${ }^{2}$ (proximal superior vena cava-right pulmonary artery anastomosis, $3 \mathrm{~mm}$ in diameter) could not be performed because of the very short proximal

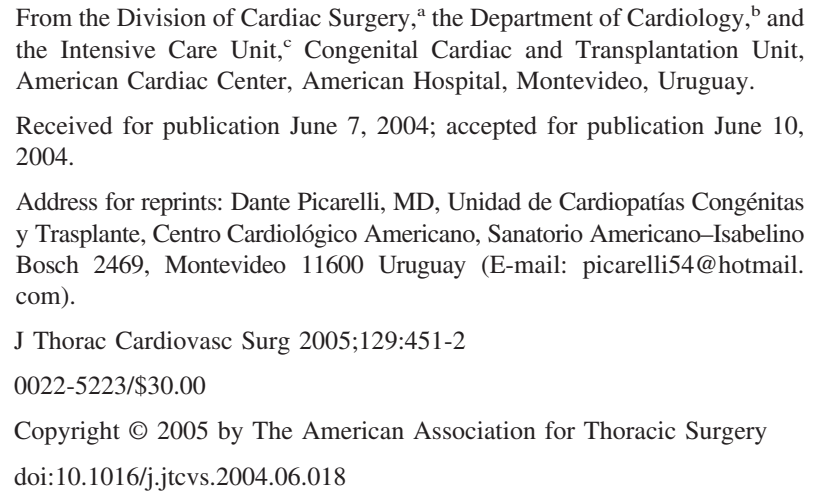

segment of the superior vena cava. We decided to construct a 3-mm anastomosis between the apex of the right atrial appendage and the inferior aspect of the right pulmonary artery (Figure 1).

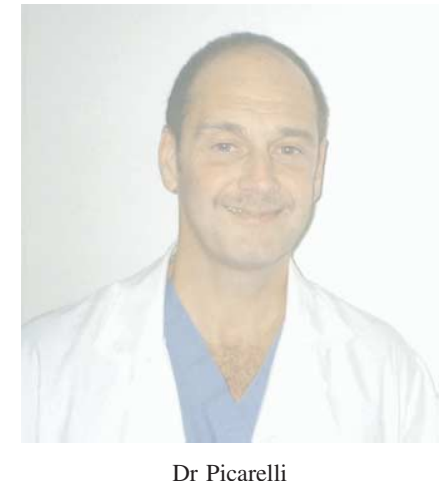

Despite a stenotic pulmonary outflow tract, banding of the pulmonary trunk was added as necessary to regulate the amount of antegrade flow required to maintain the mean pulmonary artery pressure at less than $16 \mathrm{~mm} \mathrm{Hg} .{ }^{3}$ Mean pulmonary artery pressure decreased from 20 to $14 \mathrm{~mm} \mathrm{Hg}$, with an atrial pressure of $6 \mathrm{~mm}$ $\mathrm{Hg}$ and an arterial oxygen saturation of $88 \%$.

Early postoperative echocardiography showed effective rightto-left shunt through the fenestration. Minimal inotropic support was required for 3 days. However, moderate superior vena cava syndrome, mild desaturation, and right pleural effusion developed (7 days) as a result of right diaphragmatic paralysis, which was successfully plicated. Ventilatory support was immediately discontinued, and the patient made an uneventful recovery with $88 \%$ arterial oxygen saturation.

Twelve months after the operation, the patient was in good condition, with an oxygen saturation ranging from $88 \%$ to $90 \%$, and echocardiography revealed a still open fenestration.

\section{Discussion}

Little is known about the effects of the Kawashima operation in small young infants. ${ }^{2}$ Herein we report a successful result in a 5-month-old patient with progressive cyanosis, in whom an increase of effective pulmonary blood flow was mandatory.

Despite the increasing risk of early cavopulmonary dysfunction in this age group resulting from smaller pulmonary arteries and a more reactive vascular bed, ${ }^{4}$ we decided to perform a bilateral bidirectional cavopulmonary anastomosis because of the deleterious effect of the systemic pulmonary shunt in patients with singleventricle physiology (ventricular overload and pulmonary arterial distortion). ${ }^{5}$

In an attempt to prevent the development of arteriovenous fistula, which is a risk especially in young infants with heterotaxy syndrome, ${ }^{2,3}$ we preserved forward flow from the ventricle to maintain the exposure of the pulmonary bed to hepatic flow.

To avoid cavopulmonary dysfunction caused by an additional source of pulmonary blood, we regulated the amount of the antegrade flow with pulmonary banding, maintaining the mean pulmonary artery pressure at less than $16 \mathrm{~mm} \mathrm{Hg} .{ }^{3}$ However, in this particular circulation, by adding extrapulmonary blood flow to a subtotal Fontan circulation, the concerns of having pulmonary artery and superior vena caval pressures that are too high are very 


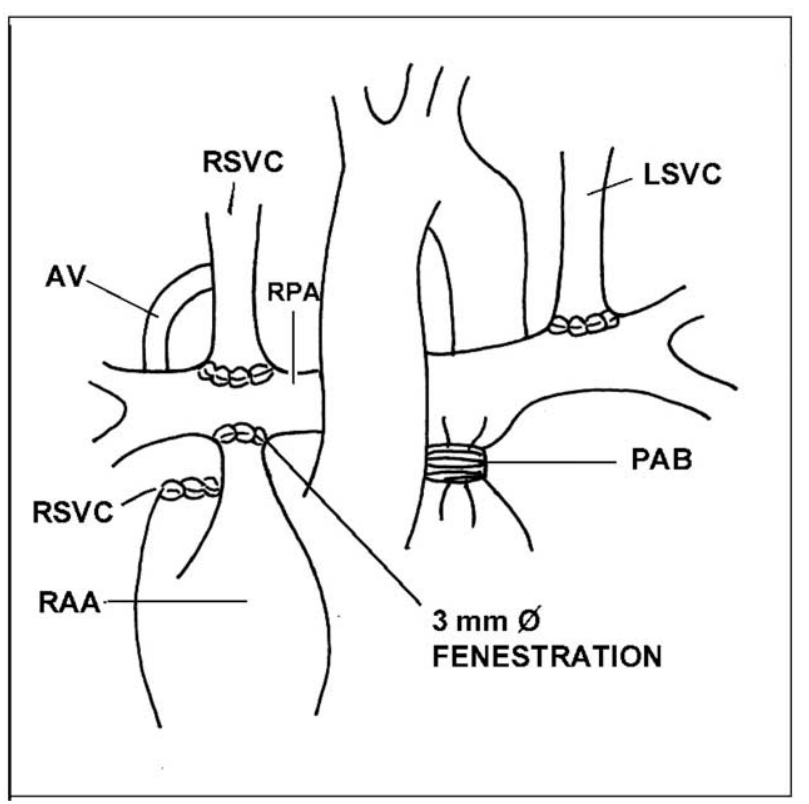

Figure 1. Modified fenestration technique for Kawashima operation in a young infant. $A V$, Azygos vein; $R S V C$, right superior vena cava; $R P A$, right pulmonary artery; $L S V C$, left superior vena cava; $R A A$, right atrial appendage; $P A B$, pulmonary artery banding.

real. ${ }^{2,3}$ This is the rationale for decompressing the pulmonary circuit by means of a limited fenestration back into the heart.

The real importance of fenestration in a small infant with this near-total Fontan circulation had been previously reported by
Hannan and associates. ${ }^{2}$ The right-to-left shunt contributed to improved hemodynamic outcome in the early postoperative period.

For anatomic reasons, the original surgical fenestration technique should be modified (Figure 1). The limited anastomosis between the apex of the right atrial appendage and the right pulmonary artery is another valuable option, avoiding the risk of sinus node injury when the proximal superior vena caval stump is too short.

Our modified surgical fenestration successfully provided an effective right-to-left shunt, as demonstrated by the good postoperative hemodynamic course, with minimal inotropic support and no significant pleural effusion, despite the potential deleterious effect of an unfortunate diaphragmatic paralysis.

We believe that the excellent outcome in our patient validates the principle of fenestration in early Kawashima operation coupled with controlled antegrade flow in young infants.

\section{References}

1. Kawashima Y, Kitamura S, Matsuda H, et al. Total cavopulmonary shunt operation in complete cardiac anomalies: a new operation. J Thorac Cardiovasc Surg. 1984;87:74-81.

2. Hannan R, Rossi A, Nykanen D, et al. The fenestrated Kawashima operation for single ventricle with interrupted inferior vena cava. Ann Thorac Surg. 2003;75:271-3.

3. Caspi J, Pettit T, Ferguson B, et al. Effects of controlled antegrade pulmonary blood flow in cardiac function after bidirectional cavopulmonary anastomosis. Ann Thorac Surg. 2003;76:1917-22.

4. Chang A, Hanley F, Wernovsky G, et al. Early bidirectional cavopulmonary shunt in young infants. Postoperative course and early results. Circulation. 1993;88:149-58.

5. Yeh T Jr, Williams W, McCrindle B, et al. Equivalent survival following cavopulmonary shunt: with or without the Fontan procedure. Eur J Cardiothorac Surg. 1999;16:111-6. 